\title{
Vortex states in mesoscopic superconducting squares: Formation of vortex shells
}

\author{
H. J. Zhao, V. R. Misko, and F. M. Peeters* \\ Department of Physics, University of Antwerpen, Groenenborgerlaan 171, B-2020 Antwerpen, Belgium \\ V. Oboznov and S. V. Dubonos \\ Institute of Solid State Physics, Russian Academy of Sciences, Chernogolovka 142432, Russia \\ I. V. Grigorieva \\ School of Physics and Astronomy, University of Manchester, Manchester M13 9PL, United Kingdom \\ (Received 9 June 2008; revised manuscript received 22 August 2008; published 17 September 2008)
}

\begin{abstract}
We analyze theoretically and experimentally vortex configurations in mesoscopic superconducting squares. Our theoretical approach is based on the analytical solution of the London equation using Green's-function method. The potential-energy landscape found for each vortex configuration is then used in Langevin-type molecular-dynamics simulations to obtain stable vortex configurations. Metastable states and transitions between them and the ground state are analyzed. We present our results of the first direct visualization of vortex patterns in micrometer-sized $\mathrm{Nb}$ squares, using the Bitter decoration technique. We show that the filling rules for vortices in squares with increasing applied magnetic field can be formulated, although in a different manner than in disks, in terms of formation of vortex "shells."
\end{abstract}

DOI: $10.1103 /$ PhysRevB.78.104517

PACS number(s): 74.25.Qt, 74.25.Ha, 74.78.Na

\section{INTRODUCTION}

The growing interest in studying vortex matter in mesoscopic and nanopatterned superconductors is closely related to recent progress in nanofabrication and perspectives of their use in nanodevices manipulating single flux quanta. As distinct from bulk superconductors, vortex states in nanoscopic and mesoscopic samples are determined by the interplay between the intervortex interaction (which is modified due to the presence of boundaries) and the confinement. In general, the shape of a mesoscopic sample is incommensurate with the triangular Abrikosov lattice, and as a consequence, the resulting vortex patterns display strong features of the sample shape and may differ strongly from a triangular lattice. Strong finite-size effects in conjunction with strong shape effects determine the vortex configurations. For example, in mesoscopic disks vortices, as shown theoretically ${ }^{1-6,9}$ and experimentally, ${ }^{7}$ form circular symmetric shells [similar to two-dimensional (2D) system of charged classical particles $\left.{ }^{8}\right]$. Moreover, due to strong confinement effects in small disks, vortices can even merge into a giant vortex $(\mathrm{GV})$, i.e., a single vortex containing more than one flux quantum, ${ }^{4}$ as was recently confirmed experimentally. ${ }^{10}$ Furthermore, it was recently demonstrated ${ }^{11}$ that vortices can merge into a cluster or a GV in micrometer-sized mesoscopic niobium disks which is induced by strong disorder in combination with rather weak confinement, while neither of these effects alone would lead to a GV/cluster formation. Similarly, shape- and symmetry-induced vortex patterns can be formed in mesoscopic superconducting triangles, ${ }^{12,14,15}$ squares, ${ }^{12,16-18}$ or, in general, in symmetric polygons. ${ }^{12,13}$ However, unlike disks where the vortex patterns result from the interplay between the discrete symmetry of the (triangular) vortex lattice and the cylindrical $\left(C_{\infty}\right)$ symmetry of the disk, mesoscopic polygons have discrete symmetry that can coincide (triangles, $C_{3}$ symmetry) or include as a subgroup (e.g., hexagons with $C_{6}$ symmetry) the symmetry of the vortex lattice. In such cases highly stable vortex configurations are possible for some values of magnetic field (providing commensurate numbers of vortices) because the vortexvortex interaction is enhanced by the effect of boundaries. Strikingly, strong boundary effects can even lead to symmetry-induced vortex states with antivortices ${ }^{14-16}$ (i.e., the symmetry of the vortex configuration with antivortices can be restored by the generation of a vortex-antivortex pair).

In contrast to $C_{3 n}$-symmetric (where $n$ is an integer) polygons, squares are incommensurate with triangular vortex lattice for any applied magnetic field. The vortex-vortex interaction and the effect of boundaries are always competing in mesoscopic squares. Resulting from this interplay: (i) the ground state of the vortex system always involves nonzero elastic energy and, as a consequence, (ii) there are metastable states with energies close to the ground state (or, in principle, the ground state even could be degenerate). Early studies on vortices in mesoscopic squares were either limited to very small samples with characteristic sizes of the order of $\xi$ (where $\xi$ is the coherence length) which were able to accommodate only few vortices, ${ }^{12}$ or they focused on the possibility of generation and stability of vortex-antivortex patterns in squares. ${ }^{16-18}$ Here we present a systematic theoretical analysis of vortex configurations in mesoscopic squares and their first direct observation in micrometer-sized niobium squares using the Bitter decoration technique. To study the formation of vortex patterns and transitions between the ground and metastable states, we analytically solve the London equation using Green's-function method, and perform moleculardynamics simulations. To obtain the stable vortex configurations, we analyze the filling of squares by vortices with increasing applied magnetic field and the formation of vortex "shells," similarly to those observed in disks.

The paper is organized as follows. The theoretical formalism and the solution of the London equation using Green's- 
function method, for a system of $L$ vortices in a rectangle sample, are described in Sec. II. In Sec. III, we discuss the evolution of vortex configurations with magnetic field calculated using the solution of the London equation found in Sec. II and the molecular-dynamics simulations (Sec. III A). We formulate the filling rules and discuss the formation of vortex shells in mesoscopic superconducting squares in Sec. III B. Metastable states and the transitions between them and the ground state are analyzed in Sec. III C. In Sec. IV, we present the results of our direct experimental observations of vortex patterns in niobium squares using the Bitter decoration technique, and compare the calculated patterns with the experimentally measured vortex configurations. The conclusions are given in Sec. V.

\section{THEORY: THE LONDON APPROACH}

We consider a strong type-II superconductor (i.e., characterized by the Ginzburg-Landau parameter $\kappa=\lambda / \xi \gg 1$, where $\lambda$ is the London penetration depth and $\xi$ is the coherence length) with rectangular cross section in the $x-y$ plane and thickness $d$ in the $z$ direction. Note that the London approach is applicable also for weak type II superconductors in case of thin-film samples with thickness $d \ll \lambda$ where the penetration depth is modified: $\lambda \rightarrow \Lambda=\lambda / d^{2}$, or in case of low vortex densities in rather large mesoscopic samples (i.e., with the lateral dimensions $a, a \gtrsim \Lambda$ ) where vortices are well separated and the order parameter is $|\Psi|^{2}=1$ everywhere except at the vortex cores. The latter case corresponds to our experiments with micrometer-sized niobium squares as described below. In our model the external magnetic field $\mathbf{H}$ is applied normal to the $x-y$ plane, i.e., along the $z$ axis: $\mathbf{h}$ $=h \mathbf{z}$. We also assume that the vortex cores are straight lines along the $z$ direction. Then the local magnetic field can be found by solving the London equation:

$$
-\lambda^{2} \nabla^{2} h+h=\Phi_{0} h \sum_{i=1}^{L} \delta\left(\mathbf{r}-\mathbf{r}_{i}\right),
$$

where $\Phi_{0}$ is the flux quantum and $\left\{\mathbf{r}_{i}=\left(x_{i}, y_{i}\right), i=1, \ldots, L\right\}$ are the positions of $L$ vortices. If we also neglect the distortion of the external magnetic field due to the sample, i.e., assume that the value of the magnetic field outside the sample near its boundary is equal to the applied field, then the boundary conditions for the magnetic field are:

$$
h( \pm a / 2, y)=h(x, 0)=h(x, b)=H .
$$

The geometry of the problem is shown in Fig. 1. Green'sfunction method for solving the London equation [Eq. (1)] with the boundary conditions [Eq. (2)] was previously used by Sardella et al. ${ }^{19}$ However, they limited themselves to the special case where one of the sides of the rectangle is much larger than the other, i.e., a stripe. Such an approximation considerably simplifies the problem but the resulting solution missed the generality (the symmetry with respect to the permutation $x \rightarrow y$ ) and thus could not be used in our case of a square: $a=b$. We seek a solution of Eq. (1) with the boundary conditions [Eq. (2)], which is valid for a rectangle with arbitrary aspect ratio $a / b$. Green's function associating with the

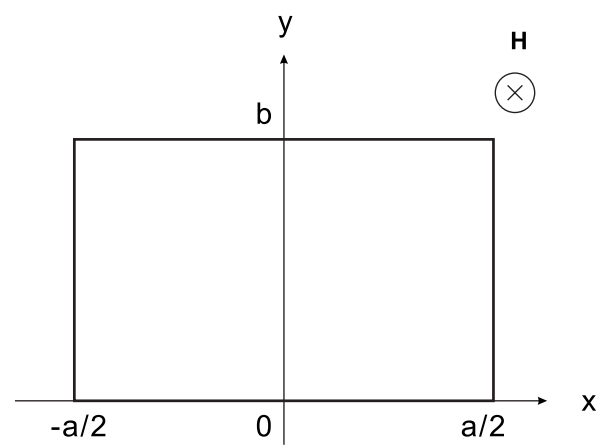

FIG. 1. The cross section of a rectangular superconductor with sides $a$ and $b$. The external magnetic field $\mathbf{H}$ is applied along the $z$ axis, and its value is assumed to be constant outside the sample.

boundary problem defined by Eqs. (1) and (2) must satisfy the following equation:

$$
-\lambda^{2} \nabla^{2} G+G=\delta\left(x-x^{\prime}\right) \delta\left(y-y^{\prime}\right),
$$

and the boundary conditions

$$
G( \pm a / 2, y)=G(x, 0)=G(x, b)=0 .
$$

Multiplying Eq. (1) by $G$ and Eq. (3) by $h$ and subtract one from another, we obtain

$-\lambda^{2}\left(G \nabla^{2} h-h \nabla^{2} G\right)=G \Phi_{0} \sum_{i=1}^{L} \delta\left(\mathbf{r}-\mathbf{r}_{i}\right)-h \delta\left(x-x^{\prime}\right) \delta\left(y-y^{\prime}\right)$.

Integrating Eq. (5) over the sample area, we arrive at

$$
\begin{aligned}
& -\lambda^{2} \int_{-a / 2}^{a / 2} d x \int_{0}^{b} d y\left(G \nabla^{2} h-h \nabla^{2} G\right) \\
& =\int_{-a / 2}^{a / 2} d x \int_{0}^{b} d y\left[G \Phi_{0} \sum_{i=1}^{L} \delta\left(\mathbf{r}-\mathbf{r}_{i}\right)-h \delta\left(x-x^{\prime}\right) \delta\left(y-y^{\prime}\right)\right] .
\end{aligned}
$$

Further we use Gauss theorem,

$$
\begin{gathered}
-\lambda^{2} \int_{-a / 2}^{a / 2} d x \int_{0}^{b} d y\left(G \nabla^{2} h-h \nabla^{2} G\right) \\
=-\lambda^{2} \oint_{\text {boundary }} d l\left(G \frac{\partial h}{\partial n}-h \frac{\partial G}{\partial n}\right),
\end{gathered}
$$

where $\partial / \partial n$ is the derivative in the normal direction to the boundary, and the boundary conditions [Eqs. (4) and (2)], and we find the expression for the magnetic field,

$$
\begin{aligned}
h\left(x^{\prime}, y^{\prime}\right)= & H\left[1-\int_{-a / 2}^{a / 2} d x \int_{0}^{b} d y G\left(x, y, x^{\prime}, y^{\prime}\right)\right] \\
& +\Phi_{0} \sum_{i=1}^{L} G\left(x_{i}, y_{i}, x^{\prime}, y^{\prime}\right) .
\end{aligned}
$$

Therefore, the problem of finding the solution for the local magnetic field is reduced to the determination of Green's 
function $G\left(x, y, x^{\prime}, y^{\prime}\right)$. In order to find a solution to Eq. (3) with the boundary condition Eq. (4), we expand Green's function in a Fourier series,

$$
G\left(x, y, x^{\prime}, y^{\prime}\right)=\frac{2}{b} \sum_{m=1}^{\infty} \sin \left(\frac{m \pi y^{\prime}}{b}\right) \sin \left(\frac{m \pi y}{b}\right) g_{m}\left(x, x^{\prime}\right) .
$$

Note that the boundary conditions [Eq. (4)] are satisfied at $y=0, b$. Further we substitute this expansion into Eq. (3) and obtain

$$
\begin{array}{r}
-\lambda^{2} \frac{2}{b} \sum_{m=1}^{\infty}\left[\frac{\partial^{2} g_{m}\left(x, x^{\prime}\right)}{\partial x^{2}} \sin \left(\frac{m \pi y^{\prime}}{b}\right) \sin \left(\frac{m \pi y}{b}\right)\right. \\
-\left(\frac{m \pi}{b}\right)^{2} g_{m}\left(x, x^{\prime}\right) \sin \left(\frac{m \pi y^{\prime}}{b}\right) \sin \left(\frac{m \pi y}{b}\right) \\
\left.+\sin \left(\frac{m \pi y^{\prime}}{b}\right) \sin \left(\frac{m \pi y}{b}\right) g_{m}\left(x, x^{\prime}\right)\right] \\
=\delta\left(x-x^{\prime}\right) \frac{2}{b} \sum_{m=1}^{\infty} \sin \left(\frac{m \pi y^{\prime}}{b}\right) \sin \left(\frac{m \pi y}{b}\right),
\end{array}
$$

where we used the following $\delta$-function representation:

$$
\delta\left(y-y^{\prime}\right)=\frac{2}{b} \sum_{m=1}^{\infty} \sin \left(\frac{m \pi y^{\prime}}{b}\right) \sin \left(\frac{m \pi y}{b}\right)
$$

since $\left\{\sqrt{\frac{2}{b}} \sin \left(\frac{m \pi y}{b}\right), \quad m=1,2,3 \ldots\right\}$ forms a complete set of orthonormal functions. As a result, we obtain the following equation for the Fourier transform of Green's function $g_{m}\left(x, x^{\prime}\right)$,

$$
-\lambda^{2} \frac{\partial^{2} g_{m}\left(x, x^{\prime}\right)}{\partial x^{2}}+\alpha_{m}^{2} g_{m}\left(x, x^{\prime}\right)=\delta\left(x-x^{\prime}\right),
$$

where

$$
\alpha_{m}=\left[1+\lambda^{2}\left(\frac{m \pi}{b}\right)^{2}\right]^{1 / 2} .
$$

The functions $g_{m}\left(x, x^{\prime}\right)$ must satisfy the boundary conditions $g_{m}\left( \pm a / 2, x^{\prime}\right)=0$. In order to solve Eq. (10), we first take its Fourier transform,

$$
-\lambda^{2}(i \omega)^{2} F(\omega)+\alpha_{m}^{2} F(\omega)=\frac{1}{2 \pi} e^{-i \omega x^{\prime}},
$$

where

$$
F(\omega)=\frac{e^{-i \omega x^{\prime}}}{2 \pi\left(\lambda^{2} \omega^{2}+\alpha_{m}^{2}\right)},
$$

from which we obtain a particular solution to Eq. (10)

$$
\begin{aligned}
\left.g_{m}\right|_{a \rightarrow \infty} & =\frac{1}{2 \alpha_{m} \lambda} e^{-\alpha_{m}\left|x-x^{\prime}\right| \lambda} \\
& =\frac{1}{2 \alpha_{m} \lambda}\left\{\cosh \left[\alpha_{m}\left(x-x^{\prime}\right) / \lambda\right]-\sinh \left(\alpha_{m}\left|x-x^{\prime}\right| \lambda\right)\right\} .
\end{aligned}
$$

The general solution of Eq. (10) reads as

$$
\begin{aligned}
g_{m}= & \frac{1}{2 \alpha_{m} \lambda}\left\{\cosh \left[\alpha_{m}\left(x-x^{\prime}\right) / \lambda\right]-\sinh \left(\alpha_{m}\left|x-x^{\prime}\right| \lambda\right)\right\} \\
& +A\left(x^{\prime}\right) \sinh \left(\alpha_{m} x / \lambda\right)+B\left(x^{\prime}\right) \cosh \left(\alpha_{m} x / \lambda\right) \\
= & \frac{1}{2 \alpha_{m} \lambda}\left[-\sinh \left(\alpha_{m}\left|x-x^{\prime}\right| \lambda\right)+C\left(x^{\prime}\right) \sinh \left(\alpha_{m} x / \lambda\right)\right. \\
& \left.+D\left(x^{\prime}\right) \cosh \left(\alpha_{m} x / \lambda\right)\right] .
\end{aligned}
$$

Using the boundary conditions [Eq. (4)] we find the coefficients $C\left(x^{\prime}\right)$ and $D\left(x^{\prime}\right)$,

$$
\begin{gathered}
C\left(x^{\prime}\right)=-\operatorname{coth}\left(\alpha_{m} a / 2 \lambda\right) \sinh \left(x^{\prime}\right) ; \\
D\left(x^{\prime}\right)=\tanh \left(\alpha_{m} a / 2 \lambda\right) \cosh \left(x^{\prime}\right) .
\end{gathered}
$$

Then the solution for $g_{m}\left(x, x^{\prime}\right)$ is given by

$$
\begin{aligned}
g_{m}\left(x, x^{\prime}\right)= & \frac{1}{2 \lambda \alpha_{m} \sinh \left(\alpha_{m} a / \lambda\right)} \\
& \times\left\{\cosh \left[\alpha_{m}\left(\left|x-x^{\prime}\right|-a\right) / \lambda\right]-\cosh \left[\alpha_{m}\left(x+x^{\prime}\right) / \lambda\right]\right\} .
\end{aligned}
$$

Inserting this result into Eq. (8), we obtain the following expression for Green's function:

$$
\begin{aligned}
G\left(x, y, x^{\prime}, y^{\prime}\right) & \\
= & \frac{2}{b} \sum_{m=1}^{\infty} \sin \left(\frac{m \pi y^{\prime}}{b}\right) \sin \left(\frac{m \pi y}{b}\right) \frac{1}{2 \lambda \alpha_{m} \sinh \left(\alpha_{m} a / \lambda\right)} \\
& \times\left\{\cosh \left[\alpha_{m}\left(\left|x-x^{\prime}\right|-a\right) / \lambda\right]-\cosh \left[\alpha_{m}\left(x+x^{\prime}\right) / \lambda\right]\right\} .
\end{aligned}
$$

From it we obtain the following expression for the local magnetic field:

$$
\begin{aligned}
h(x, y)= & \Phi_{0} \sum_{i=1}^{L} G\left(x_{i}, y_{i}, x, y\right)+H\left\{\frac{\cosh [(y-b / 2) / \lambda]}{\cosh (b / 2 \lambda)}\right. \\
& +\frac{4}{b} \sum_{m=0}^{\infty} \frac{b}{\alpha_{2 m+1}^{2}(2 m+1) \pi} \sin \left[\frac{(2 m+1) \pi y}{b}\right] \\
& \left.\times \frac{\cosh \left(\alpha_{2 m+1} x / \lambda\right)}{\cosh \left(\alpha_{2 m+1} a / 2 \lambda\right)}\right\} .
\end{aligned}
$$

Note that this solution is valid for a rectangle with arbitrary aspect ratio $a / b$ and is a generalization of the earlier result presented in Ref. 19.

Using the obtained solution or the London equation for the local distribution of the magnetic field $h(x, y)$, we obtain the Gibbs free energy per unit length of an arbitrary vortex configuration, 


$$
\begin{aligned}
\mathcal{G}= & \sum_{i=1}^{L}\left(\epsilon_{i}^{\text {shield }}+\sum_{j=1}^{L} \epsilon_{i j}^{v}\right)+\epsilon^{\text {core }}+\epsilon^{\text {field }} \\
= & \frac{\Phi_{0} H}{4 \pi A} \sum_{i=1}^{L}\left\{\frac{\cosh \left[\left(y_{i}-b / 2\right) / \lambda\right]}{\cosh (b / 2 \lambda)}+\frac{4}{b} \sum_{m=0}^{\infty} \alpha_{2 m+1}^{-2} \frac{b}{(2 m+1) \pi} \sin \left[\frac{\left.(2 m+1) \pi y_{i}\right]}{b}\right] \frac{\cosh \left(\alpha_{2 m+1} x_{i} / \lambda\right)}{\cosh \left(\alpha_{2 m+1} a / 2 \lambda\right)}\right\} \\
& +\frac{\Phi_{0}^{2}}{8 \pi A} \sum_{i=1}^{L} \sum_{j=1}^{L} G\left(x_{i}, y_{i}, x_{j}, y_{j}\right)-\frac{H^{2}}{8 \pi}\left\{\frac{\tanh (b / 2 \lambda)}{b / 2 \lambda}-\frac{8}{\pi^{2}} \sum_{m=0}^{\infty} \frac{\tanh \left(\alpha_{2 m+1} a / 2 \lambda\right)}{\left[(2 m+1) \alpha_{2 m+1}\right]^{2}\left(\alpha_{2 m+1} a / 2 \lambda\right)}\right\}-L \frac{\Phi_{0} H}{4 \pi A} .
\end{aligned}
$$

Here, $A=a \times b$ is the area of the rectangle. The last two terms are the energies associated with the external magnetic field and the vortex cores, respectively. Green's function in the first term describes the interaction between vortices and also the interaction between vortices and their images, which are situated outside the sample. The second term represents the interaction between the $i$ th vortex and the shielding currents. Note that in Ref. 19, the authors limited their consideration to the case of a thin film such that $(\pi \lambda / b)^{2} \gg 1$ and the term "1" in Eq. (11) can be neglected. The London theory has a singularity for the interaction between a vortex and its own image (self-interaction). We notice that when $i=j$ Green's function does not converge. To avoid divergency, we apply a cutoff procedure (see, e.g., Refs 20-22), which means a replacement of $\left|\mathbf{r}_{i}-\mathbf{r}_{j}\right|$ by $a \xi$ for $i=j$. It was shown in Ref. 23 that the results of the London theory agree with those of the Ginzburg-Landau theory, the vortex size should be chosen as $\sqrt{2} \xi$, and therefore we take $a=\sqrt{2}$. The confinement energy is given by $\epsilon_{c}=\epsilon_{i}^{\text {shield }}+\epsilon_{i i}$.

In Figs. 2(a) and 2(b), we plot the distribution of the confinement energy for mesoscopic squares with $a=3 \lambda$ and $a$ $=15 \lambda$, correspondingly. In the mesoscopic square with $a$ $=3 \lambda$, Fig. 2(a), the screening current extends inside the square and interacts with all the vortices. But in the large mesoscopic square (we call it "macroscopic") with $a=15 \lambda$, only the vortices which are close to the boundary feel the screening current. In the mesoscopic square, vortices strongly overlap with each other [see Fig. 2(c)], while in the macroscopic square, the interaction between vortices is rather weak and only the closest neighbors are important [see Fig. 2(d)]. This difference between small (mesoscopic) and large (macroscopic) squares leads, in general, to the size dependence of the vortex patterns in mesoscopic samples as it was recently demonstrated for disks (see Ref. 9).

\section{THE EVOLUTION OF VORTEX PATTERNS WITH MAGNETIC FIELD}

\section{A. Molecular-dynamics simulations of vortex patterns}

Within the London approach, vortices can be treated as pointlike "particles," and it is convenient to employ molecular dynamics (MD) for studying the vortex motion driven by external forces (see, e.g., Refs. 9, 11, 24, and 25), similarly to a system of classical particles. ${ }^{8}$ In Sec. II we obtained the analytic expression for the free energy of a system of $L$ vor- tices as a function of the applied magnetic field [Eq. (15)]. The force felt by the $i$ th vortex can be obtained by taking the derivative of the energy in the following:

$$
F_{i}=-\nabla_{i} \mathcal{G},
$$

where $\nabla_{i}=\frac{\partial}{\partial x_{i}} \mathbf{e}_{x}+\frac{\partial}{\partial y_{i}} \mathbf{e}_{y}$ is the two-dimensional derivative operator.

The overdamped equation of vortex motion can be presented in the following form:

$$
\eta \mathbf{v}_{i}=\mathbf{F}_{i}=\sum_{j \neq i} \mathbf{F}_{i j}+\mathbf{F}_{\text {self }}^{i}+\mathbf{F}_{M}^{i}+\mathbf{F}_{T}^{i}
$$

where the first three terms are as follows: $\mathbf{F}_{i j}$ is the force due to the repulsive vortex-vortex interaction of the $i$ th vortex with all other vortices, $\mathbf{F}_{\text {self }}^{i}$ is the interaction force with the image, and $\mathbf{F}_{M}^{i}$ is the force of interaction with the external magnetic field which enters the sample through the boundaries; $\eta$ is the viscosity, which is set here to unity. Note that Eq. (16) contains these three terms [with the free energy defined by Eq. (15)], and in Eq. (17) we added a thermal stochastic term $\mathbf{F}_{T}^{i}$ to simulate the process of annealing in the experiment. The thermal stochastic term should obey the following conditions:

$$
\left\langle F_{i}^{T}(t)\right\rangle=0
$$

and

$$
\left\langle F_{i}^{T}(t) F_{i}^{T}\left(t^{\prime}\right)\right\rangle=2 \eta k_{B} T \delta_{i j} \delta\left(t-t^{\prime}\right) .
$$

It is convenient to express the lengths in units of $\lambda$, the fields in units of $H_{c 2}$, the energies per unit length in units of $g_{0}$ $=\Phi_{0}^{2} / 8 \pi A \cdot 1 / \lambda^{2}$, and the force per unit length in units of $f_{0}$ $=\Phi_{0}^{2} / 8 \pi A \cdot 1 / \lambda^{3}$, where $A$ is the sample's area. In our calculations we use the value of the Ginzburg-Landau parameter $\kappa=6$ taken from the experiment with $\mathrm{Nb}$ (see below).

In order to find the ground-state vortex configurations in squares, we perform stimulated annealing simulations by numerically integrating the overdamped equations of motion Eq. (17). The procedure is as follows. First we generate a random vortex distribution and set a high value of temperature. Then we gradually decrease the temperature to zero, i.e., simulating the annealing process in real experiments (see, e.g., Ref. 26). To find the minimum-energy configuration, we perform many simulation runs with random initial distributions and count the statistics of the appearance of different vortex configurations for each $L$. This procedure 


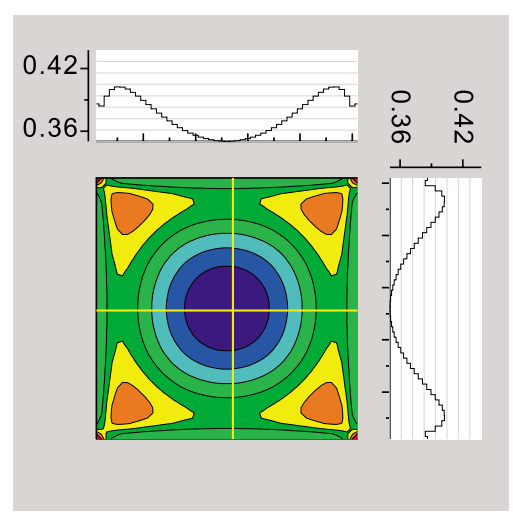

(a)
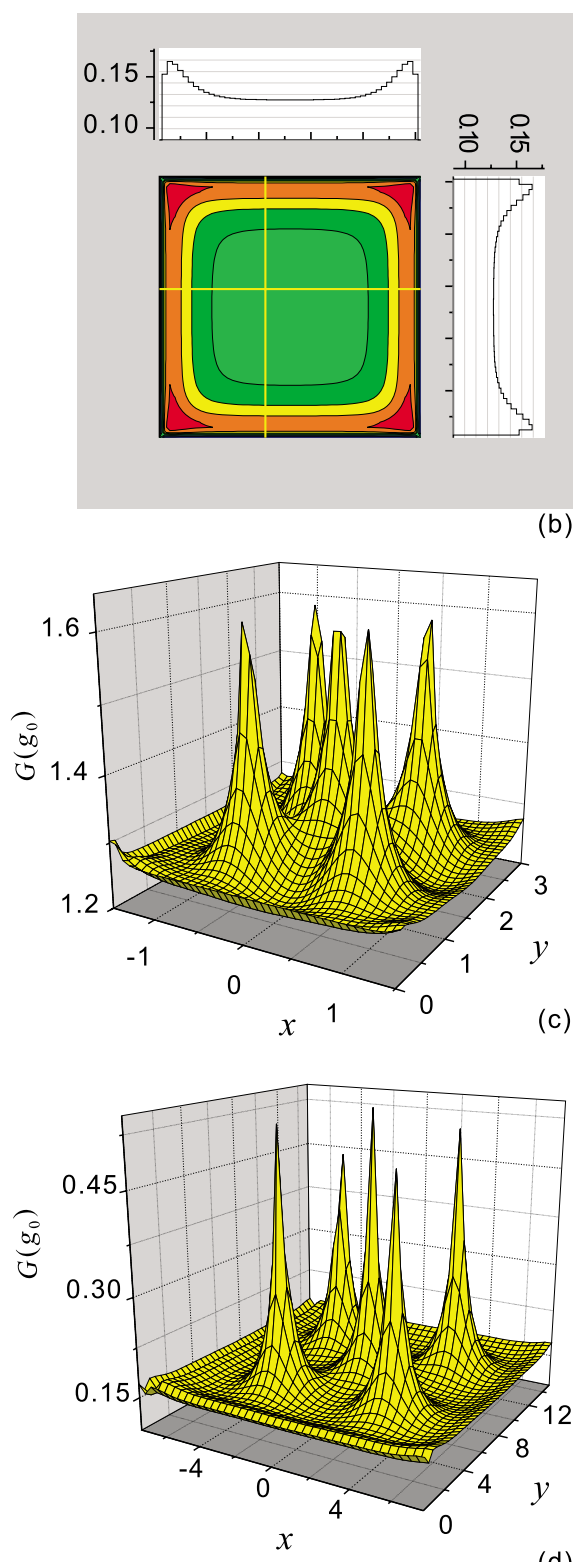

(d)

FIG. 2. (Color online) The profiles of the confinement energy $\epsilon_{c}=\epsilon_{i}^{\text {shield }}+\epsilon_{i i}$ (measured in units of $g_{0}=\Phi_{0}^{2} / 8 \pi A \cdot 1 / \lambda^{2}$, where $A$ is the area of the sample) for mesoscopic superconducting squares with size (a) $a=3 \lambda$ and (b) $15 \lambda$. The Gibbs free-energy distributions for squares with (c) $a=3 \lambda$ and (d) $15 \lambda$ for the vortex state with $L=5$.
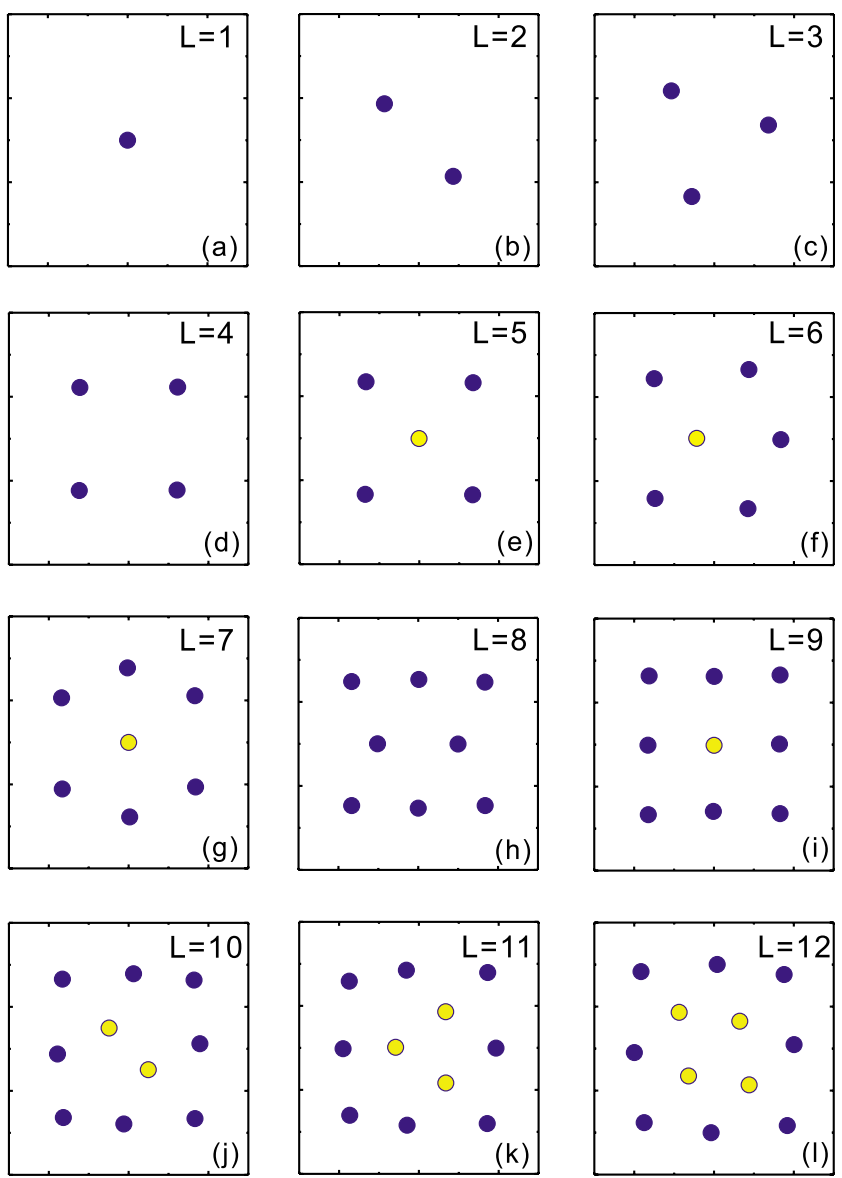

FIG. 3. (Color online) The evolution of vortex configurations for the states with vorticity increasing from $L=1$ to 12 , in a superconducting square with $a=3 \lambda$ (the same results found for larger squares, e.g., with $a=15 \lambda$ ). The vortices in the outer shell are shown by the blue (black) circles while the inner-shell vortices are shown by the yellow (gray) circles. The formation of the second shell starts when $L=5$.

simulates $^{9}$ the statistical analysis of experimental data with simultaneous measurements of vortex configurations in arrays of many (up to 300) practically identical samples. It was used in experiments with $\mathrm{Nb}$ disks in Refs. 7 and 11 and also in experiments with $\mathrm{Nb}$ squares presented in this paper.

\section{B. Filling rules for vortices in squares with increasing magnetic field: Formation of vortex shells}

The results for the vortex patterns for different vorticities $L$ are shown in Figs. 3 and 4. With increasing applied magnetic field, vortex configurations evolve as follows: Starting from a Meissner state with no vortex, the first vortex appears in the center [see Fig. 3(a)], for $L=2$ the two are located symmetrically on the diagonal [see Fig. 3(b)]. Further increase of the magnetic field leads to the formation of a triangular vortex pattern having a common symmetry axis with the square, which is the diagonal [see Fig. 3(c)]. For $L=4$ vortices arrange themselves in a perfect square, Fig. 3(d), whose symmetry is commensurate with the sample and therefore it turns out that this is a highly stable vortex 

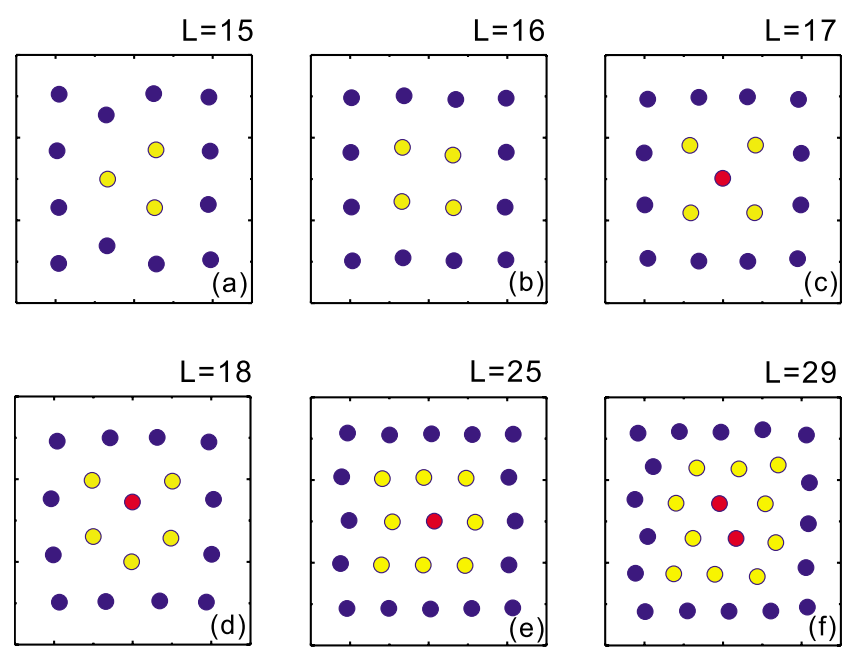

FIG. 4. (Color online) The evolution of vortex configurations for $L=15-18$ (a)-(d), and for (e) $L=25$ to (f) 29 , in a superconducting square with $a=3 \lambda$. For vorticities [(a)-(d)] $L=15-18$, the outermost shell formed by 12 vortices is complete (commensurate with the square boundary), and with increasing magnetic-field vortices fill inner shells. Note that when the inner shell also becomes complete $[$ (b) $L=16$, state $(4,12)]$, the third shell starts to form for (c) $L=17$. For states with larger vorticities, e.g., (e) $L=25$ and (f) $L$ $=29$, the vortex patterns are very close to a triangular lattice which is distorted near the boundary.

configuration. ${ }^{5,27}$ Note that even in the bulk the gain in the elastic energy is very small during the transition from the triangular vortex lattice to the square one, and consequently, in the presence of a square boundary, it turns out that a square vortex lattice can be easily stabilized (for commensurate vortex numbers). For vorticity $L=5$, vortices tend to form either a pentagon, or a square with one vortex in the center [see Fig. 3(e); the transition between this configuration and the pentagonlike pattern will be discussed below]. The additional vortex appears in the center thus forming a second shell in a similar way as in disks, $, 6,7,9$ but in the latter, this occurred for a larger $L$-value $(L=6)$. To distinguish different shells and indicate the number of vortices in each shell, we use the same notations as in Refs. 6, 7, and 9. For example, the pentagonlike configuration and the pattern with four vortices in the outer shell and one vortex in the center are denoted as $(5)$ and $(1,4)$, respectively. (It is clear that vortex shells in squares are not as well defined as in disks and sometimes it is a matter of choice how to define them.) Compared with disks, which have $C_{\infty}$ symmetry, the $C_{4}$ symmetry of squares induces a new element of symmetry in the resulting vortex patterns. In other words, vortex patterns in squares (tend to) acquire elements of the $C_{4}$ symmetry even if they are not arranged in a perfect square lattice. For example, the calculated vortex patterns share one $[L=6$, Fig. $3(\mathrm{f})]$ or two $[L=7$ and 8 , Figs. $3(\mathrm{~g})$ and $3(\mathrm{~h})$, correspondingly] symmetry axes of the square parallel to its side. This tendency to share symmetry elements with the square boundary remains also for larger vorticities as can be seen, e.g., in Figs. 3(j)-3(1) for vorticities $L=10,11$, and 12, respectively. For the commensurate number of vortices $L=9$, a perfect symmetric square-lattice pattern is formed.
Using the concept of vortex shells, we analyzed the filling rules for mesoscopic superconducting squares with increasing magnetic field. To summarize these rules, for $L=1$ to 4 , vortices are arranged in a single shell, the second shell appears when $L=5$, and then vortices fill the shells as follows: As the vorticity $L$ increases from $L=5$ to 9 , the new vortices fill the outer shell. Then the number of vortices in the inner shell starts to increase for $L \geq 9$ [see Figs. 3(j)-3(1)]. This occurs because the outer shell is formed by eight vortices (i.e., three per each side) which turns out to be stable. Thus, the new vortices fill the inner shell until $L=12$. Then, again, the newly generated vortices start to fill the outermost shell until $L=16$, when the number of vortices in the outermost shell becomes 12 , which is also stable (i.e., commensurate with the square boundary). The formation of the third shell starts when the vorticity becomes $L=17$ [note that for $L$ $=17$ the vortices can arrange themselves either in a two-shell configuration $(5,12)$ or in a three-shell configuration $(1,4,12)$, which occurs to have a slightly lower energy, see analysis below]. In a similar way, the filling of shells occurs for larger values of $L$ (e.g., for 3-, 4-shell patterns, etc.). As a general rule, the outermost shells containing $4 N$ vortices, where $N$ is an integer, are very stable. With increasing the density of vortices, the average distance between them decreases. As a result, the interaction between vortices becomes more and more important leading to the formation of the triangularlattice phase away from the boundary. Therefore, the triangular lattice is recovered for large vorticities being distorted near the square boundaries. Note that for large enough $L$ vortices do not form a square lattice even for commensurate vortex numbers (e.g., for $L=25,36$, etc.) as it does for $L$ $=4,9$, and 16. Some examples of two- and three-shell vortex patterns are shown in Fig. 4.

\section{The ground state and metastable states}

The incommensurability of the square boundary with the triangular vortex lattice creates metastable vortex configurations. While in many cases metastable states are well separated in energy from the ground state, in some cases, namely, for borderline configurations having $n$ and $n+1$ shells, the lowest-energy metastable state can become almost indistinguishable from the ground state. In such cases, vortex states with very close energies can have comparable probability to be realized experimentally. An example of such a state is the case $L=5$. The stable states for $L=5$ are shown in the insets of Fig. 5. In order to examine which one is more stable, we investigate the free energy as a function of the displacement of one of the vortices while we allow the other vortices to relax to their lowest-energy positions. We start with the pentagonlike configuration (5) (the left inset) and we change the position of this vortex moving it toward the center of the square and let the other vortices adjust their positions accordingly. At the end, we arrive at the square-symmetric state $(1,4)$. We plot the free energy of the system as a function of the displacement of this vortex from its equilibrium position, and we repeat this procedure for all the vortices A, B, C, D, and $\mathrm{E}$ (we always move only one vortex while all others relax to minimize the free energy). For any of the five vor- 

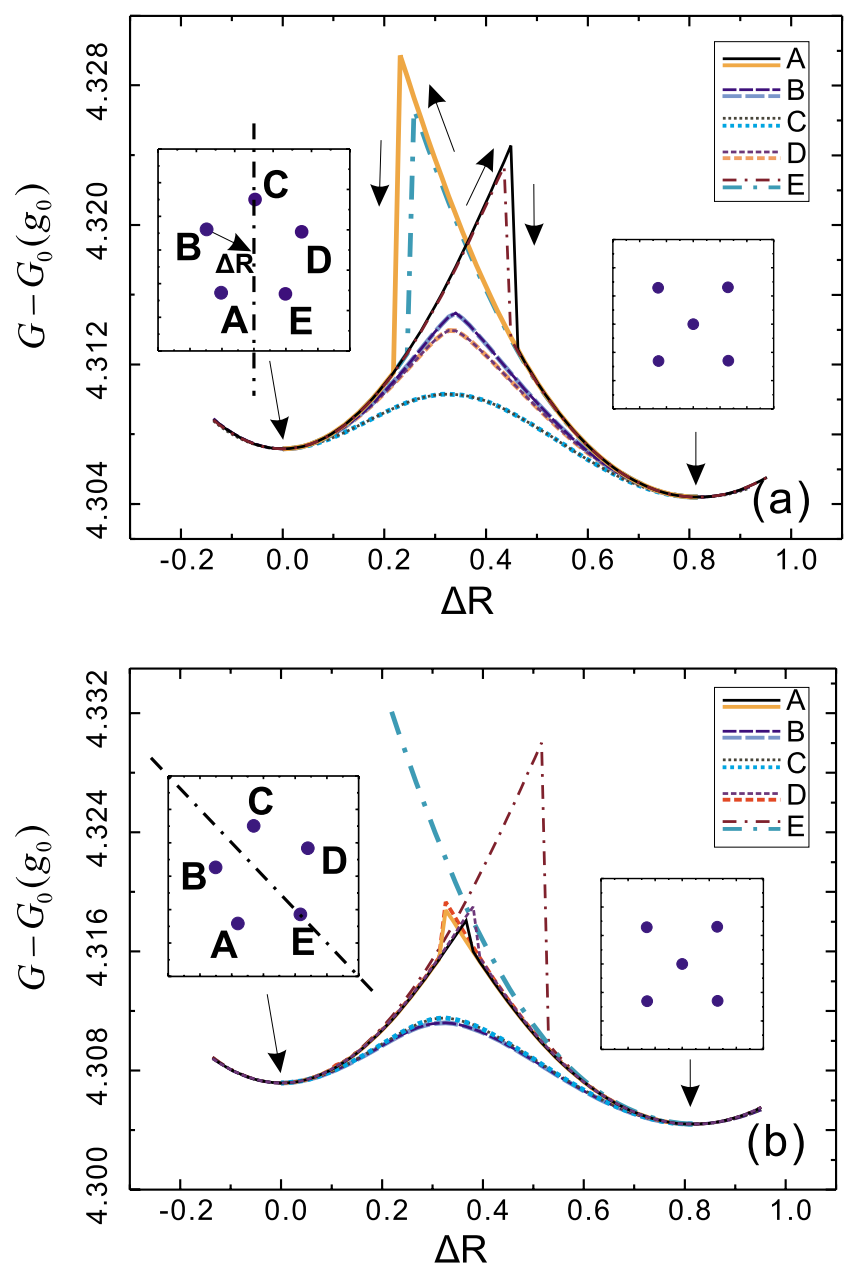

FIG. 5. (Color online) The change of the free energy $\left(G-G_{0}\right)$ versus the displacement $R$ of one of the vortices in the initial pentagon-shaped configuration from its initial position toward the center [two different lines for each configuration correspond to increasing and decreasing $\Delta R$ as shown by the arrows in (a)]. $G_{0}$ is the free energy associated with external magnetic field and the vortex cores [term " 4 " in Eq. (15)], which is independent of the positions of the vortices. The two stable states, the pentagonlike state (5) and the square-symmetric state $(1,4)$, are shown in the insets. The vortices are labeled by A, B, C, D, and E. Two different symmetry axes of the configuration (5) are shown by the dash-dotted line in the insets of (a) and (b), respectively. The side of the square is $a=3 \lambda$. In both cases, the configuration with one vortex in the center $(1,4)$ has a lower energy than the pentagonlike pattern $(5)$. Note that the curves for B and D (and for A and E) are slightly different due to the fact that the configuration (5) is not perfectly aligned with respect to the symmetry axes.

tices, this procedure leads to a barrier between the two states. We notice that there are two possible pentagonlike configurations (5) which share different symmetry axes with the square, see Figs. 5(a) and 5(b). The difference of their free energy is less than $10^{-4}$. In Fig. 5(a) we see that the motion of vortex $\mathrm{C}$ is accompanied with the lowest-energy barrier. This is because vortices $\mathrm{A}, \mathrm{B}, \mathrm{D}$, and $\mathrm{E}$ are already close to their final positions in state $(1,4)$. Moving vortex B or D leads to a higher energy barrier. Finally, moving vortex A or $\mathrm{E}$ to the center is associated with the highest barrier and

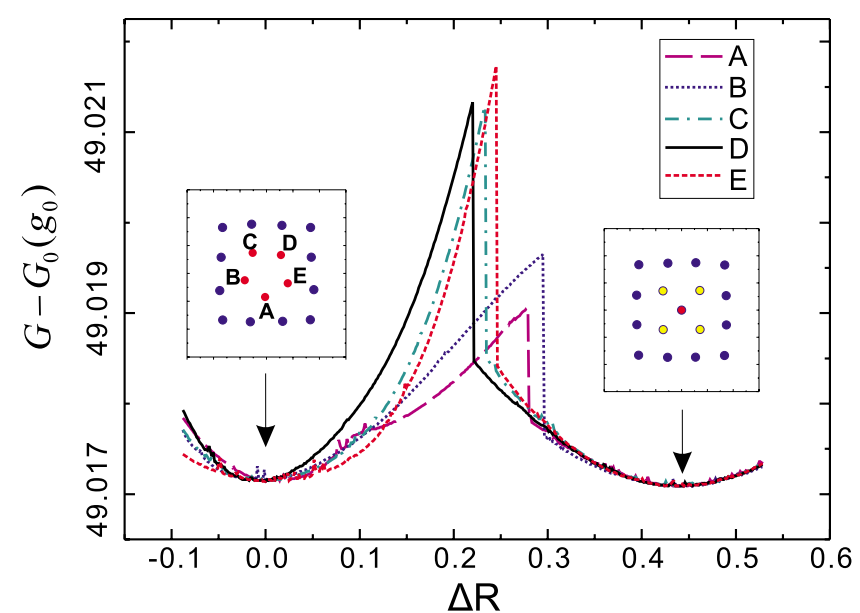

FIG. 6. (Color online) The change of the free energy $\left(G-G_{0}\right)$ versus the displacement $R$ of one of the vortex in the inner shell of the state $(5,12)$ from its initial position toward the center; $G_{0}$ is defined in the caption of Fig. 5. The change in the free energy due to the movement of the vortices in the inner shells [i.e., $(5,12) \rightarrow$ $(1,4,12)]$ is damped by the movement of the vortices in the outermost shell which act as a "softer" wall than the boundary [in the case of the transition $(5) \rightarrow(1,4)$, see Fig. 5]. The movement of the vortices in the outmost shell causes more saddle points. The two states, $(5,12)$ and $(1,4,12)$, have very close free energies.

passing over a saddle point (jump in $G-G_{0}$ ). Then we move the central vortex of state $(1,4)$ back to its initial positions in state (5). The highest-barrier transitions (i.e., curves A and E) show a hysteretic behavior which is an indication of metastable states.

In Fig. 5(b), we show the results of the calculation of the free energy as a function of displacement of a vortex, for a different modification of the state (5), i.e., when the vortex configuration has the symmetry axis coinciding with the diagonal of the square [cf. Fig. 5(a)]. Note that these two configurations of state (5) have practically the same free energy and thus equal probability to appear in experiment. Moving vortex $\mathrm{E}$, which is situated on the diagonal of the square [see the left inset in Fig. 5(b)], is accompanied by the highest energy barrier compared to moving other vortices. The reverse process (i.e., moving the central vortex to position $\mathrm{E}$ ) leads to a very high potential barrier, and the pentagonlike state cannot be restored unless a random (thermal) force is added to break the symmetry. Moving vortex $\mathrm{B}$ or $\mathrm{C}$ is accompanied by the lowest-energy barrier. State $(1,4)$ has a lower free energy than state (5). According to our calculations, it is the ground state for $L=5$.

Similar transitions are found between two- and three-shell vortex configurations for $L=17$ (see Fig. 6). Twelve vortices form the outermost shell and the other five can form either a one-shell or two-shell configurations similarly as state $L=5$. Again, we move one of the five vortices in the inner shell of the state $(5,12)$ to the center of the square. The analysis of the free energy shows that the difference of the free energy between the two states $\left(|\Delta G| \sim 10^{-5}\right)$ is much smaller compared to the states for $L=5\left(|\Delta G| \sim 10^{-3}\right)$. The reason for this is that for $L=17$, the twelve vortices in the outermost shell can adjust themselves to lower the free energy, which create 
much "softer" walls for the five vortices in the inner shell than the sample boundary. Thus, the change of the free energy due to the movement of the vortices in the inner shells can be more or less compensated by the movement of the vortices in the outermost shell.

\section{EXPERIMENTAL OBSERVATION OF VORTEX CONFIGURATIONS IN MESOSCOPIC NB SQUARES}

To visualize the corresponding vortex configurations experimentally we used the well-known Bitter decoration technique which is based on in situ evaporation of 10-20 nm Fe particles that are attracted to regions of magnetic field created by individual vortices and thus allow their visualization (details of the technique are described elsewhere ${ }^{28}$ ). The mesoscopic samples for this study were made from a $150 \mathrm{~nm}$ thick $\mathrm{Nb}$ film deposited on a $\mathrm{Si}$ substrate using magnetron sputtering. The film's superconducting parameters were: transition temperature $T_{c}=9.1 \mathrm{~K}$; magnetic-field penetration depth $\lambda(0) \approx 90 \mathrm{~nm}$; coherence length $\xi(0) \approx 15 \mathrm{~nm}$; and upper critical field $H_{c 2}(0) \approx 1.5 \mathrm{~T}$. Using e-beam lithography and dry etching with an Ar ion beam through a $250 \mathrm{~nm}$ thick Al mask, the films were made into arrays of small square "dots" of four different sizes, with the side of the square, $a$, varying from 1 to $5 \mu \mathrm{m}$. Each array typically contained 150-200 such dots. A whole array was decorated in each experiment, allowing us to obtain a snapshot of up to 100 vortex configurations in dots of the same shape and size, produced in identical conditions (same applied magnetic field $H$ and temperature $T$, same decoration conditions). It was therefore possible to simultaneously visualize vortex configurations for several different vorticities $L$ (in samples of different sizes) and also to gain enough statistics for quantitative analysis of the observed vortex states in terms of their stability, sensitivity to sample imperfections, and so on. Below we present the results obtained after field cooling to $T \approx 1.8 \mathrm{~K}$ in perpendicular external fields ranging from $H$ $=20$ to $60 \mathrm{Oe}$. We note that the above temperature $(1.8 \mathrm{~K})$ represents the starting temperature for the experiments. Thermal evaporation of Fe particles usually leads to a temporary increase in temperature of the decorated samples but the increase never exceeded $2 \mathrm{~K}$ in the present experiments, leaving the studied $\mathrm{Nb}$ dots in the low-temperature limit, $T$ $<0.5 T_{c}$.

Figure 7 shows examples of vortex configurations observed for vorticities $L=2-13$. The images shown in Fig. 7 were obtained in several different experiments and on samples of different sizes (see figure caption). We note that the same vorticity $L$ could be obtained for different combinations of the applied field and the size of the square, e.g., $L=6$ was found for $H=60 \mathrm{Oe}, a=2 \mu \mathrm{m}$, and $H=40$ Oe, $a$ $=2.5 \mu \mathrm{m}-$ see images in Figs. 8(b) and 7(e), respectively. Sometimes two different vorticities were found in the same experiment for nominally identical squares, e.g., both $L=9$ and $L=10$ were found for $H=35$ Oe and $a=3.5 \mu \mathrm{m}-$ see images in Figs. 7(g)-7(i). The latter finding can be explained by slightly different shapes of individual squares or by an extra vortex captured during field cooling; see Ref. 7 for a more detailed discussion, where the same effect was found

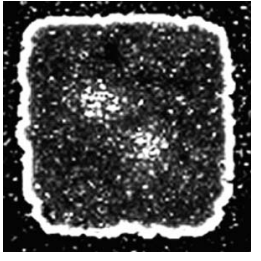

(a)

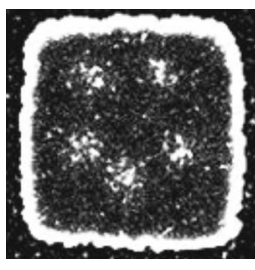

(d)

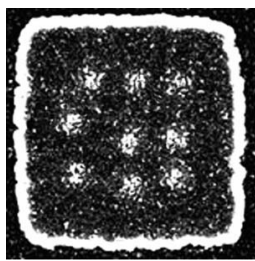

(g)
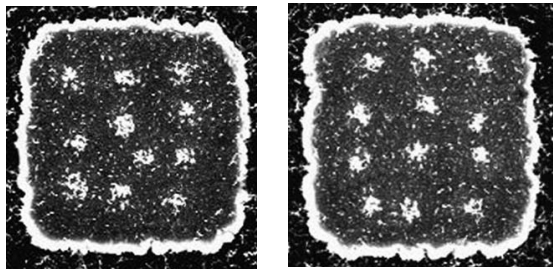

(k)

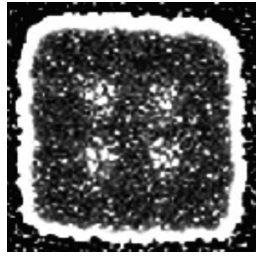

(c)
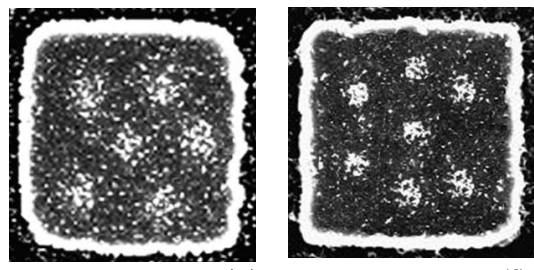

(e)

(f)

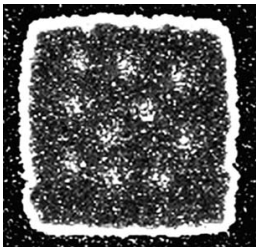

(h)
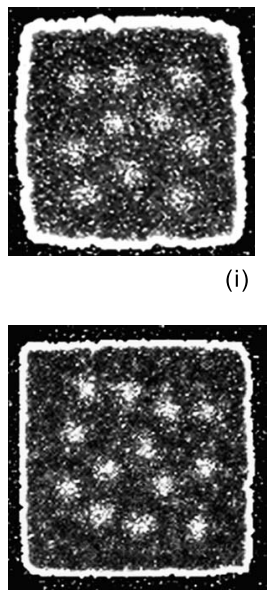

(I)
FIG. 7. Scanning electron microscope (SEM) images of vortex configurations observed experimentally for vorticities $L=2-13$. Vortex positions are indicated by clusters of small white $(\mathrm{Fe})$ particles. (a) $L=2$; sample size (side of the square) $a \approx 2.5 \mu \mathrm{m}, H$ $=20 \mathrm{Oe}$; (b) $L=3 ; a \approx 2 \mu \mathrm{m}, H=35 \mathrm{Oe}$; (c) $L=4 ; a \approx 2.4 \mu \mathrm{m}$, $H=40 \mathrm{Oe} ; \quad$ (d) $L=5 ; \quad a \approx 2.4 \mu \mathrm{m}, H=40 \mathrm{Oe} ;$ (e) $L=6 ; a$ $\approx 2.5 \mu \mathrm{m}, H=40 \mathrm{Oe} ;(\mathrm{f}) L=7 ; a \approx 2 \mu \mathrm{m}, H=60 \mathrm{Oe} ;(\mathrm{g}) L=9$; $a \approx 3.5 \mu \mathrm{m}, H=35 \mathrm{Oe}$; (h) $L=10 ; a \approx 3.5 \mu \mathrm{m}, H=35$ Oe; (i) $L$ $=10 ; a \approx 3.5 \mu \mathrm{m}, H=35 \mathrm{Oe} ;(\mathrm{j}) L=11 ; a \approx 2.5 \mu \mathrm{m}, H=60 \mathrm{Oe}$; (k) $L=12 ; a \approx 2.6 \mu \mathrm{m}, H=60 \mathrm{Oe} ; \quad(1) L=13 ; a \approx 5 \mu \mathrm{m}, H$ $=20$ Oe.

for circular mesoscopic disks. Overall, the vorticity as a function of the applied field $H$ showed the same behavior as that found earlier for circular disks, ${ }^{7}$ i.e., the square dots showed strong diamagnetic response for small vorticities $L$ $<10$ (also observed earlier in disks with a strong disorder ${ }^{11}$ ) while for larger vorticities the extra demagnetization per vortex saturated at $\delta \Phi / \Phi \approx 0.2$, in excellent agreement with earlier numerical studies. ${ }^{12}$

Most of the vortex configurations shown in Fig. 7 represent just one of several possible states for each vorticity [with the exception of images (h) and (i) which both correspond to $L=10]$. Indeed, for most vorticities we found more than one well-defined vortex configuration and some of these were found with almost the same probability, indicating that, in agreement with theory described above, vortices in meso- 

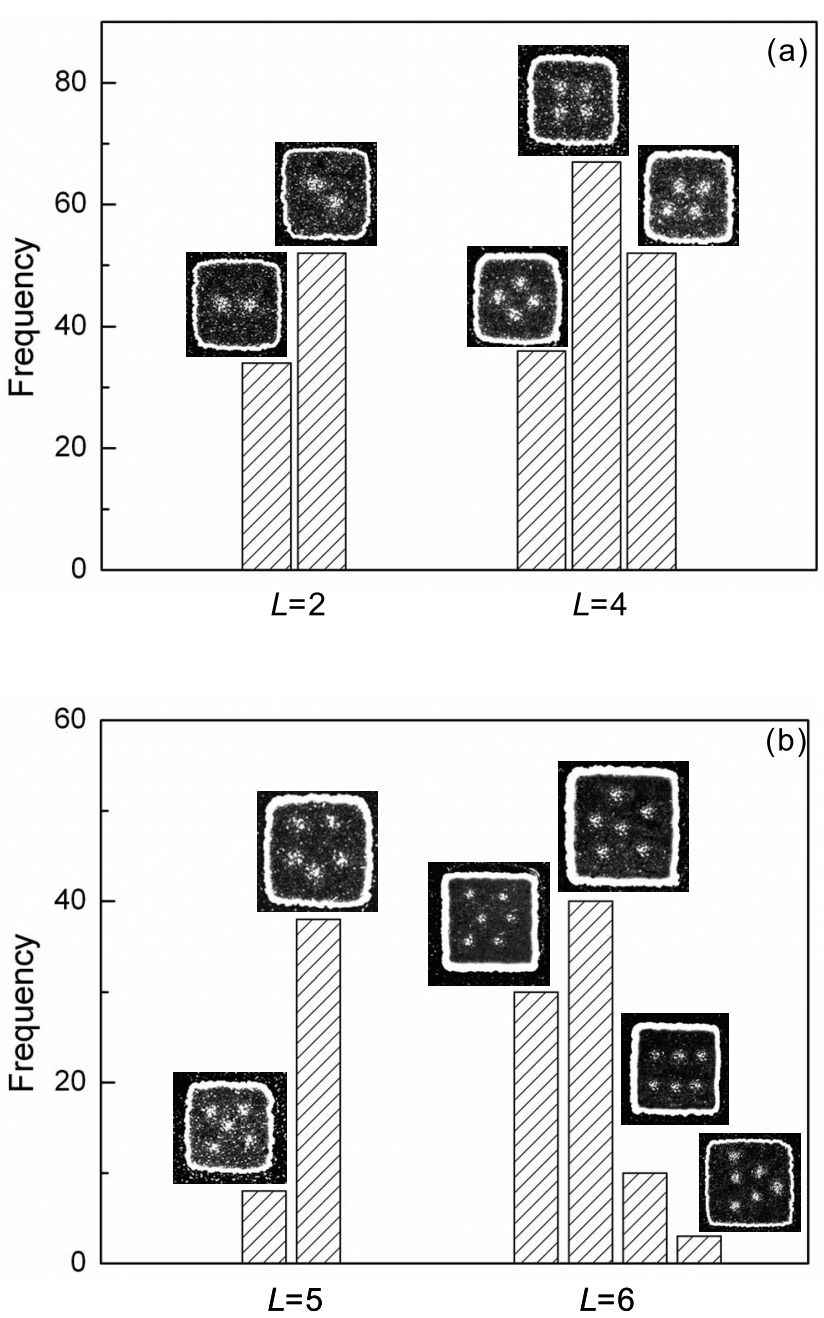

FIG. 8. Histograms of different vortex states observed for vortices $L=2,4$ (for squares with $a=2 \mu \mathrm{m}$ ) (a) and $L=5$ (for squares with $a=2 \mu \mathrm{m})$ and 6 (b) $(a=2 \mu \mathrm{m}$ and $a=2.5 \mu \mathrm{m})$. SEM images of the corresponding vortex configurations are shown as insets.

scopic squares form not only the ground, but also metastable states, and the energies of the latter are often very close to the energy of the ground state. This conclusion follows from our statistical analysis of all observed vortex configurations which resulted in histograms such as those shown in Fig. 8 for $L=2,4,5$, and 6 . For $L=2$ and 4 , the most frequently observed states agree with the ground states found theoretically [see Figs. 3(b) and 3(d)] and the metastable states appear to have similar energies, as they are found with similar probabilities. As expected, both states for $L=2$ and two of the states for $L=4$ have vortices sitting along the symmetry axes of the square, with the diagonal axis being slightly preferable. The third state for $L=4$ [on the right-hand side in Fig. $8(\mathrm{a})]$ is more unusual in that the vortices are sitting in the apexes of a rhombus that is slightly rotated with respect to the diagonal of the square. Although this particular state did not come out in the numerical simulations, ${ }^{29}$ it was found with a high probability in experiment and, moreover, the rhombus-based vortex configurations were also found for larger vorticities both in experiment [see, e.g., Fig. 7(1) for $L=13$ ] and theory [see rhombic inner shells for $L=12$ and 16 in Figs. 3(1) and 4(b), respectively].
For $L=6$, one of the two most frequently observed states [also shown in Fig. 7(e)] corresponds exactly to the ground state found numerically [Fig. 3(f)] but the state found in experiment with the highest probability is the more symmetric two-shell configuration with the outer shell having the same pentagon shape as that found for $L=5$. This $L=6$ state can be viewed as a direct precursor of the two-shell states for $L=7$ and 9, which were found as ground states both in theory [Figs. 3(g) and 3(i)] and experiment [Figs. 7(f) and 7(g)]. For $L=5$, two possible states - a two-shell configuration with one vortex in the center $(1,4)$ and four vortices in the corners and a pentagonlike configuration (5)-were found in experiment and in numerical simulations. However, numerical simulations found a slightly lower energy for the two-shell configuration $(1,4)$ (see Fig. 5), while in experiment the pentagonshaped configuration was found to appear more frequently. This discrepancy is unlikely to be related to the nonideal character of the experimental squares: As we show below, neither the roughness of the boundaries, nor the presence of some pinning in the experimental samples have any noticeable effect on the observed vortex configurations, due to strong confinement (see, e.g., Fig. 2). It is possible that, due to the very small difference in free energies between the two states (which becomes practically negligible for samples with $a \gg \lambda$ ), the vortex configurations for $L=5$ are particularly sensitive to the exact sample size (in experiment the squares are almost ten times larger than in the analysis of Fig. 5). The sensitivity of vortex configurations to sample size was studied in detail for circular disks (see Ref. 9) and was indeed found to affect the stability of some (but not all) vortex states. For higher vorticities, $L=7-13$, we found welldefined two-shell configurations most of which correspond to the stable configurations found numerically. The outer shell in these configurations was either square [see Figs. $7(\mathrm{~g})-7(\mathrm{k})$ for $L=9-12]$, circular $[L=7$, Fig. $7(f)]$ or rhombic $[L=13$, Fig. 7(1)] with vortices of the inner shell either sitting along one of the symmetry axes of the square, as for $L=2$, or forming a triangle, as for $L=3$. For certain matching vorticities ( $L=9$ and 12), the observed two-shell configurations correspond to a square vortex lattice.

We note that the irregularities of the sample shape and uneven boundaries of some of our dots have, surprisingly, no discernible effect on the observed configurations of vortices (i.e., the vortices form regular, symmetric patterns). For example, the dots in Figs. 7(j) and 7(k) have especially rounded corners and very rough boundaries but the vortex configurations have square symmetries. Similarly, the same $L=6$ state was found in dots with rounded corners, as in Fig. 7(e), and in almost perfect squares, as in the image shown in Fig. 8(b). Furthermore, we found that for a given value of $L$ the observed configurations did not depend on the sample size or the applied field, at least within the studied field range-see Fig. 9 for an example.

Finally, we compared the experimentally observed positions of vortices within the square dots with those found numerically and found an excellent agreement, as demonstrated by Fig. 9. Here we show a superposition of theoretical images from Fig. 3 and experimental images for the same vortex configurations. Two of the images [Figs. 9(d) and $9(\mathrm{e})]$ compare the same theoretical configuration with experi- 


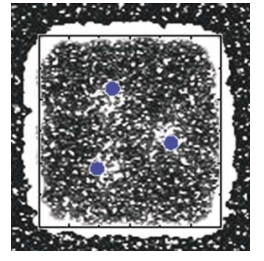

(a)

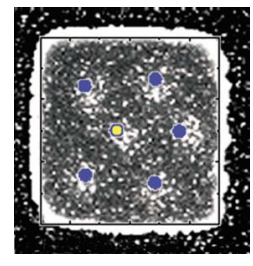

(d) (b)

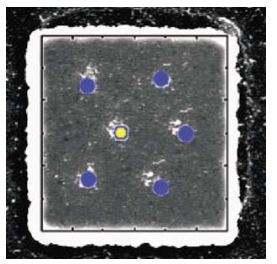

(e)

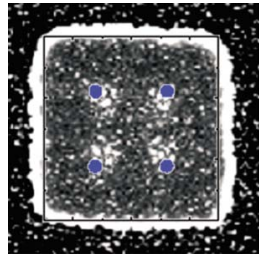

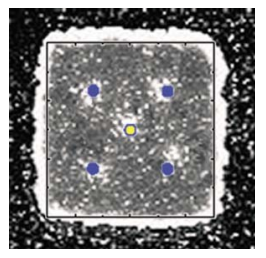

(c)

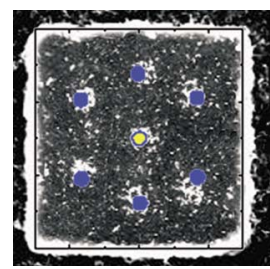

(f)
FIG. 9. (Color online) Comparison of the experimentally observed positions of vortices within the square dots with those found numerically. Superimposed on the experimental images are vortex configurations shown in Figs. 3(c)-3(g). Two experimental images for $L=6[(\mathrm{~d})$ and (e)] are superimposed on the same theoretical image [Fig. 3(f)], to demonstrate that the observed configurations did not depend on the sample size or the applied field [for image (d) $H=40$ Oe, $a \approx 2.5 \mu \mathrm{m}$, for image (e) $H=60 \mathrm{Oe}, a \approx 2 \mu \mathrm{m}]$.

mental images obtained on dots of different sizes in different applied fields $(H=40$ Oe, $a=2.5 \mu \mathrm{m}$ and $H=60$ Oe and $a$ $=2 \mu \mathrm{m}$, respectively) illustrating the point made above that the vortex configurations do not depend on the sample size and/or applied field.

Overall, despite the inevitable presence of some disorder in our samples, which was not taken into account in the calculations, there is a very good agreement between the observed vortex configurations and the calculated vortex patterns. The main features of the vortex states revealed by experiment is formation of vortex shells with predominantly square symmetry for vorticities $L \geq 7$ and vortex patterns following the main symmetry axes of the square for small vorticities $L \leq 4$. The two intermediate vorticities $L=5$ and 6 appear to be a special case: Here the mismatch between the square shape of the dot and the natural symmetry of the vortex lattice is more difficult to accommodate and the preferred vortex configurations turned out to be the pentagon- shaped shell for $L=5$ and three different patterns for $L=6$, none of which has the fourfold symmetry of the square.

\section{CONCLUSIONS}

We performed a systematic study of vortex configurations in mesoscopic superconducting squares and compared the results with vortex patterns observed experimentally in micrometer-sized $\mathrm{Nb}$ squares using the Bitter decoration technique.

In the theoretical analysis we relied upon the analytical solution of the London equation in mesoscopic squares by using Green's-function method and the image technique. The stable vortex configurations were calculated using the technique of molecular-dynamics simulations simulating the stimulated annealing process in experiments.

We revealed the filling rules for squares with growing number of vortices $L$ when gradually increasing the applied magnetic field. In particular, we found that for small $L$ vortices tend to form patterns that are commensurate with the symmetry of the square boundaries of the sample. The filling of "shells" (similar to mesoscopic disks) occurs by periodic filling of the outermost and internal shells. With increasing vorticity, the outermost shell is filled until it is complete (i.e., the number of vortices in it becomes $4 N$, where $N$ is an integer, i.e., commensurate with the square boundary). Then vortices fill internal shells until the number of vortices becomes large enough to create the outermost shell with $4(N$ $+1)$ vortices. Again, after that vortices fill internal shells. With increasing vorticity, the shell structure becomes less pronounced, and for large enough $L$ the vortex patterns in squares becomes a triangular lattice distorted near the boundaries.

\section{ACKNOWLEDGMENTS}

We thank Mauro M. Doria for useful discussions. This work was supported by the Flemish Science Foundation (FWO-Vl), the Interuniversity Attraction Poles (IAP) Programme-Belgian State-Belgian Science Policy, the "Odysseus" program of the Flemish Government and FWO-V1, and EPSRC (U.K.). V.R.M. is funded by the EU Marie Curie project under Contract No. MIF1-CT-2006-040816.

\footnotetext{
*francois.peeters@ua.ac.be

${ }^{1}$ A. K. Geim, I. V. Grigorieva, S. V. Dubonos, J. G. S. Lok, J. C. Maan, A. E. Filippov, and F. M. Peeters, Nature (London) 390, 259 (1997).

${ }^{2}$ P. S. Deo, V. A. Schweigert, F. M. Peeters, and A. K. Geim, Phys. Rev. Lett. 79, 4653 (1997).

${ }^{3}$ V. A. Schweigert and F. M. Peeters, Phys. Rev. B 57, 13817 (1998).

${ }^{4}$ V. A. Schweigert and F. M. Peeters, Phys. Rev. Lett. 83, 2409 (1999).

${ }^{5}$ B. J. Baelus, F. M. Peeters, and V. A. Schweigert, Phys. Rev. B 63, 144517 (2001).
}

${ }^{6}$ B. J. Baelus, L. R. E. Cabral, and F. M. Peeters, Phys. Rev. B 69 , 064506 (2004).

${ }^{7}$ I. V. Grigorieva, W. Escoffier, J. Richardson, L. Y. Vinnikov, S. Dubonos, and V. Oboznov, Phys. Rev. Lett. 96, 077005 (2006).

${ }^{8}$ V. M. Bedanov and F. M. Peeters, Phys. Rev. B 49, 2667 (1994).

${ }^{9}$ V. R. Misko, B. Xu, and F. M. Peeters, Phys. Rev. B 76, 024516 (2007).

${ }^{10}$ A. Kanda, B. J. Baelus, F. M. Peeters, K. Kadowaki, and Y. Ootuka, Phys. Rev. Lett. 93, 257002 (2004).

${ }^{11}$ I. V. Grigorieva, W. Escoffier, V. R. Misko, B. J. Baelus, F. M. Peeters, L. Y. Vinnikov, and S. V. Dubonos, Phys. Rev. Lett. 99, 147003 (2007). 
${ }^{12}$ B. J. Baelus and F. M. Peeters, Phys. Rev. B 65, 104515 (2002).

${ }^{13}$ L. F. Chibotaru, A. Ceulemans, G. Teniers, and V. V. Moshchalkov, Physica C 369, 149 (2002).

${ }^{14}$ L. F. Chibotaru, A. Ceulemans, V. Bruyndoncx, and V. V. Moshchalkov, Nature (London) 408, 833 (2000); Phys. Rev. Lett. 86, 1323 (2001).

${ }^{15}$ V. R. Misko, V. M. Fomin, J. T. Devreese, and V. V. Moshchalkov, Phys. Rev. Lett. 90, 147003 (2003).

${ }^{16}$ R. Geurts, M. V. Milošević, and F. M. Peeters, Phys. Rev. B 75, 184511 (2007).

${ }^{17}$ A. S. Mel'nikov, I. M. Nefedov, D. A. Ryzhov, I. A. Shereshevskii, V. M. Vinokur, and P. P. Vysheslavtsev, Phys. Rev. B 65, 140503(R) (2002).

${ }^{18}$ J. Bonča and V. V. Kabanov, Phys. Rev. B 65, 012509 (2001).

${ }^{19}$ E. Sardella, M. M. Doria, and P. R. S. Netto, Phys. Rev. B 60, 13158 (1999).

${ }^{20}$ A. A. Abrikosov, Fundamentals of the Theory of Metals (NorthHolland, Amsterdam, 1986).

${ }^{21}$ A. I. Buzdin and J. P. Brison, Phys. Lett. A 196, 267 (1994).

${ }^{22}$ J. B. Ketterson and S. N. Song, Superconductivity (Cambridge
University Press, Cambridge, England, 1999).

${ }^{23}$ L. R. E. Cabral, B. J. Baelus, and F. M. Peeters, Phys. Rev. B 70, 144523 (2004).

${ }^{24}$ F. Nori, Science 271, 1373 (1996); C. Reichhardt, C. J. Olson, and F. Nori, Phys. Rev. B 57, 7937 (1998); Phys. Rev. Lett. 78, 2648 (1997); Phys. Rev. B 58, 6534 (1998).

${ }^{25}$ V. Misko, S. Savel'ev, and F. Nori, Phys. Rev. Lett. 95, 177007 (2005); V. R. Misko, S. Savel'ev, and F. Nori, Phys. Rev. B 74, 024522 (2006)

${ }^{26}$ K. Harada, O. Kamimura, H. Kasai, T. Matsuda, A. Tonomura, and V. V. Moshchalkov, Science 274, 1167 (1996).

${ }^{27}$ B. J. Baelus, A. Kanda, N. Shimizu, K. Tadano, Y. Ootuka, K. Kadowaki, and F. M. Peeters, Phys. Rev. B 73, 024514 (2006).

${ }^{28}$ I. V. Grigorieva, Supercond. Sci. Technol. 7, 161 (1994).

${ }^{29} \mathrm{~A}$ rhombus-shaped vortex state is not consistent with the $C_{4}$-symmetry and it does not appear as a metastable state in a perfect square for $L=4$. However, the presence of imperfections (or other vortices as, e.g., outer-shell vortices for states with $L$ $=12$ and 16) favors appearance of triangular- or rhombus-shaped vortex configurations. 